\title{
Predicting bladder cancer responses to PD-L1 inhibitors? A case report and overview for the busy clinician
}

\author{
Seranio N, Malkowicz SB, Aguarin L, Dorsey JF, Christodouleas J and Kao GD* \\ Departments of Radiation Oncology and Urology, University of Pennsylvania School of Medicine, Philadelphia, PA, USA
}

\section{Introduction}

Bladder cancer is the sixth most common form of cancer in the United States and ranks fourth among men. 81,190 new cases of bladder cancer and 17,240 deaths attributable to bladder cancer are predicted to occur in 2018 in the United States alone [1]. Around the world, bladder cancer is responsible for 165,000 deaths a year [2]. Muscle-invasive bladder cancer (MIBC) represents approximately 25\% of patients diagnosed with bladder cancer and carries a significant risk of death that has not significantly changed in decades [3].

Until the advent of immunotherapy, arguably the last major therapeutic development in the management of metastatic bladder cancer was platinum-based chemotherapy 30 years ago [4]. Programmed cell death protein 1 (PD-1) and programmed cell death ligand 1 (PDL1) have been identified as negative immunoregulatory molecules that promote immune evasion of tumor cells. Novel immune checkpoint inhibitors function from the blockade of PD-1/PD-L1 which ultimately enhances the anti-tumor capacity of the immune system [5]. As of the beginning of this year, there have been five therapies targeting the PD-1/ PD-L1 axis approved for second-line treatment of metastatic urothelial cancer with two approved for first-line therapy in cisplatin-ineligible patients (Tables 1 and 2).

While the rapid development of novel immunotherapies is exciting and promising, there is a need for reliable biomarkers to assist in treatment stratification. Although immune checkpoint blockade has shown encouraging results in the clinic, only a fraction of patients responds to the treatment [6]. The development of accurate patient selection methods based on effective biomarkers may have the potential to robustly treat some patients while saving others from costly interventions and unnecessary adverse events. This review presents the current evidence regarding biomarkers associated with various responses to PD-1/PD-L1 inhibitors in bladder cancer. Additionally, we present case report of a potential way to complement and bolster current biomarkers using circulating tumor cells.

\section{Predictors of response}

\section{PD-L1 overexpression}

Bladder cancer is a highly immunogenic malignancy [7]. Most of the work in exploring a biomarker to predict the anti-PD-1/PD$\mathrm{L} 1$ response has been in PD-L1 (B7-H1) expression [8]. PD-L1 is an immunoinhibitory molecule mainly expressed on the surface of tumor cells and antigen-presenting cells in various solid malignancies that suppresses the activation of cytotoxic $\mathrm{T}$ cells, leading to the progression of tumors. Overexpression of PD-L1 in bladder cancer is associated with poor clinical outcomes which makes the rationale for immunotherapy in these tumors enticing [9].

In a recent meta-analysis across a range of solid tumors, PD-L1 positivity was associated with significantly higher overall response [10]. These data therefore seem support the role of PD-L1 expression as a predictive biomarker of clinical benefit with PD-1/PD-L1 inhibitors. However, there are a number of relative differences between the various clinical trials exploring the association of PD-L1 expression in bladder cancer and overall clinical outcome. Some trials such as the study for durvalumab showed a strong association of good outcomes with higher PD-L1 expression, while in contrast the association was more muted in the Keynote-045 (pembrolizumab), IMVigor Cohort 2 (atezolizumab), or Checkmate-275 (nivolumab) studies [11-14].

It should be noted that discordant studies may be due to the use of different assays with different cut-off values for immunohistochemistry or different methods of analysis [15]. Manual correction may be needed, e..g., a recent study comparing a number of these immunoassays showed highly concordant results when a standardized manual scoring method was used despite the differences in antibody design, molecular properties, detection systems, and staining platforms [16]. A major

Table 1. FDA approved immunotherapies for bladder cancer

\begin{tabular}{|c|c|c|c|c|}
\hline Drug Name & Clinical Trial & Date Approved & $\begin{array}{l}\text { FDA Approved } \\
\text { Indication }\end{array}$ & ORR (\%) \\
\hline \multirow{2}{*}{ Atezolizumab } & \begin{tabular}{|c|} 
IMvigor210 \\
Cohort 2 (Phase \\
II)
\end{tabular} & May 18, 2016 & $\begin{array}{l}\text { 2nd line therapy } \\
\text { of mUC }\end{array}$ & 15.0 \\
\hline & \begin{tabular}{|} 
IMvigor210 \\
Cohort 1 (Phase \\
II)
\end{tabular} & April 18, 2017 & $\begin{array}{l}\text { 1st line (Cis- } \\
\text { ineligible } \\
\text { Patients) }\end{array}$ & 23.0 \\
\hline \multirow[b]{2}{*}{ Pembrolizumb } & $\begin{array}{l}\text { Keynote-045 } \\
\text { (Phase III) }\end{array}$ & May 8, 2017 & $\begin{array}{l}\text { 2nd line therapy } \\
\text { of mUC }\end{array}$ & 21.1 \\
\hline & $\begin{array}{l}\text { Keynote-052 } \\
\text { (Phase II) }\end{array}$ & May 18, 2017 & $\begin{array}{l}\text { 1st line (Cis- } \\
\text { ineligible } \\
\text { Patients) }\end{array}$ & 28.6 \\
\hline Nivolumab & $\begin{array}{c}\text { CheckMate 275 } \\
\text { (Phase II) }\end{array}$ & February 2, 2017 & $\begin{array}{c}\text { 2nd line therapy } \\
\text { of mUC }\end{array}$ & 17.6 \\
\hline Durvalumb & $\begin{array}{c}\text { MEDI4736-1108 } \\
\text { (Phase I/II) }\end{array}$ & May 1, 2017 & $\begin{array}{c}\text { 2nd line therapy } \\
\text { of mUC }\end{array}$ & 17.8 \\
\hline Avelumb & $\begin{array}{l}\text { JAVELIN } \\
\text { (Phase I) }\end{array}$ & May 8, 2017 & $\begin{array}{c}\text { 2nd line therapy } \\
\text { of mUC }\end{array}$ & 19.6 \\
\hline
\end{tabular}

${ }^{*}$ Correspondence to: Gary D Kao, MD, PhD, Departments of Radiation Oncology and Urology, University of Pennsylvania School of Medicine, Philadelphia, USA, E-mail: Gary.Kao@uphs.upenn.edu

Received: February 08, 2019; Accepted: February 25, 2019; Published: February 27, 2019 
Table 2. Biomarkers for predicting response, resistance, and hyperprogression to PD-L1 inhibitors

\begin{tabular}{|c|c|c|c|}
\hline Type of Predictor & Biomarker & Advantages & Disadvantages \\
\hline \multirow{4}{*}{ Predictors of Response } & PD-L1 Overexpression & $\begin{array}{l}\text {-The most studied biomarker } \\
\text {-Has prognostic and predictive value } \\
\text {-FDA approved assays for each therapy }\end{array}$ & $\begin{array}{l}\text {-IHC looks at a single time point } \\
\text {-Can be missed if expression is focal } \\
\text {-Lack of standardisation between assays }\end{array}$ \\
\hline & Tumor Mutational Burden & $\begin{array}{l}\text {-Clear benefits for patients with high TMB } \\
\text {-Patients with MMRD are particularly } \\
\text { susceptible }\end{array}$ & $\begin{array}{l}\text {-Large tumour heterogenicity } \\
\text {-Threshold effect of therapeutic benefit } \\
\text {-Variance between gene panels }\end{array}$ \\
\hline & TCGA Subtyping & $\begin{array}{l}\text {-Distinct signatures based on sequencing data } \\
\text {-Cluster seem to show therapeutic advantage } \\
\text { in some cases }\end{array}$ & $\begin{array}{l}\text {-Classification not yet standardized } \\
\text {-New classification scheme is not well } \\
\text { studied } \\
\text {-Performance differs between drugs }\end{array}$ \\
\hline & IDO & $\begin{array}{l}\text {-Preliminary trial results show clear benefit to } \\
\text { combination therapy }\end{array}$ & $\begin{array}{l}\text {-No studies examining the role of IDO } \\
\text { expression on response }\end{array}$ \\
\hline \multirow{2}{*}{ Predictors of Resistance } & JAK $1 / 2$ & -Appears to predict acquired resistance & -Larger studies are necessary \\
\hline & Beta-2 Microglobulin & -Appears to predict acquired resistance & -Larger studies are necessary \\
\hline Predictors of hyperprogression & MDM2 Family & $\begin{array}{l}\text {-Associated with patients who Progress } \\
\text { rapidly on immunotherapy }\end{array}$ & -Exact mechanism still needs to be elucidated \\
\hline
\end{tabular}

source for discrepancy can result from the tissue compartments that are probed for PD-L1. It has been shown that PD-L1 expression in both the tumor cell (TC) and the immune cell (IC) contribute to the inhibition of antitumor immunity and both may prove predictive of response [17]. The studies with pembrolizumab and nivolumab trials assessed for PD-L1 expression on TC, and the atezolizumab study-based PD-L1 expression on IC, while the durvalumab study used a composite of the two. The relative importance of these respective tissue compartments may be dependent on the specific solid tumor type as well as the particular drug being used.

Another limitation of PD-L1 IHC is the difficulty in distinguishing membranous from cytoplasmic staining [18]. Several studies have demonstrated that only cell membrane-expressed PD-L1 has biological significance [19]. Therefore, it may be more reasonable to analyze correlations between membrane $\mathrm{PD}-\mathrm{L} 1$ protein, rather than intracellular PD-L1 protein or mRNA, and clinical outcomes. Furthermore, PD-L1 has limited binding sites for IHC detection antibodies, as it contains only two small hydrophilic regions, making immunohistochemical approaches classically used in formalin-fixed, paraffin-embedded (FFPE) specimens less effective. Because of this lack of binding sites on PD-L1 amenable for IHC detection, IHC antibodies may bind PDL1 at structurally unique sites compared with where therapeutic PD-L1 antibodies bind [8].

There are also important spatial considerations when assessing PDL1 expression in cancers. Few studies have explored PD-L1 expression status comparing the primary lesions versus metastatic lesions. One such study appears to show a high concordance between PD-L1 expression in matched primary and metastatic urothelial carcinomas with agreement in $89.9 \%$ cases [16]. However, the majority of these matched cases were obtained from patients with regional metastases excised at the time of primary tumor resection. The expression of PDL1 can occur in two patterns, focal expression and diffuse expression [20]. Even from the same sample, a false negative biopsy may result due to the focal nature of PD-L1 expression in many tumors.

Finally, a theoretical clinical concern may be that specific tumors may down-regulate expression of PD-L1. This mechanism may facilitate cloaking against protective immune-surveillance, perhaps as a component of becoming metastatic. For example, the cell line T24, derived from a patient with grade 3 locally advanced transitional cell bladder cancer expresses PD-L1 and the cell surface protein RhoGDI2 which has been shown to suppress bladder cancer metastasis via reduction of inflammation in the tumor microenvironment.
Theodorescu et al. developed the T24T cell line, a tumorigenic variant of T24. T24T cells when injected into mice, shows a markedly increased propensity for rapid growth, metastasis, and lethality. Numerous groups have investigated the T24T cell line to better understand the mechanisms underlying its behavior [21-24]. It is intriguing to hypothesize that the aggressive and lethal nature of T24T cells may be due in part to the down-regulation of PD-L1 and other immunomodulatory factors (see western blot shown in the Figure 1).

\section{IDO}

IDO, also known as IDO1 is an enzyme encoded by the INDO gene that is principally involved in the catabolism of tryptophan to kynurenine. Tryptophan depletion is a mechanism of immunoregulation and can lead to active suppression of T-cell function [25]. IDO expression has been reported in several human cancers and has usually been associated with a worse prognosis [26].

The phase I/II KEYNOTE-037/ECHO-202 study evaluates the combination of pembrolizumab and the IDO1-inhibitor epacadostat for safety, tolerability, and efficacy in patients with select malignancies, including bladder cancer. The study shows preliminary objective response rate (ORR) and disease control rate (DCR) of 35\% and $53 \%$ respectively [27]. In another study looking at nivolumab with a selective, once-daily oral IDO1 inhibitor BMS-986205 the ORR and DCR were $32 \%$ and $44 \%$ respectively [28]. This study also looked at ORR by PD-L1 expression levels. Patients who expressed PD-L1 $>1 \%$ had an ORR that was $46 \%(n=13)$ while patients with PD-L1 $<1 \%$ had an ORR of $22 \%(n=9)$.

In contrast, ECHO-301/KEYNOTE-252, a phase 3 randomized, double-blind, placebo-controlled study evaluating KEYTRUDA in combination with epacadostat or placebo in patients with unresectable or metastatic melanoma reported disappointing results. In a press release it was revealed the study did not meet the primary endpoint of improving progression-free survival in the overall population compared to KEYTRUDA monotherapy. The study's second primary endpoint of overall survival was also unlikely to reach statistical significance. Such contrasting results await reconciliation from future studies, perhaps with better patient selection, such as including PD-L1 and IDO expression patterns in selecting patients for study. .

\section{Mutational burden}

The mutation load or tumor mutational burden (TMB) is an emerging biomarker for response to immunotherapy [29]. The 


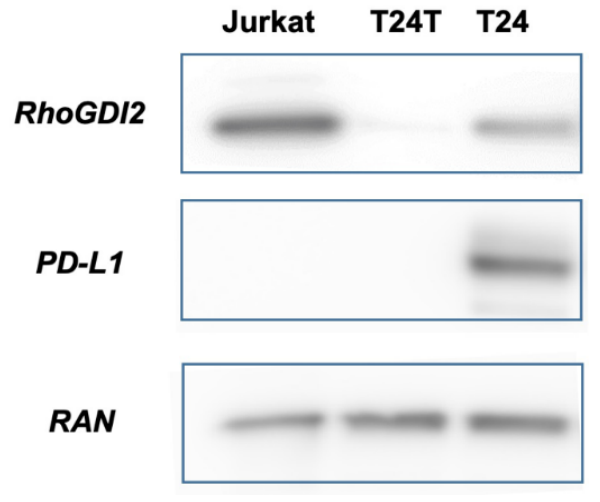

Figure 1. Western blots comparing T24 and T24T bladder cancer cell lines. The more aggressive and tumorigenic T24T shows a loss of PD-L1 expression. Jurkat cells serve as a negative control for PD-L1. RAN is a nuclear protein that serves as a loading control for all lanes

hypothesis is that tumors that are highly mutated are more likely to generate neoantigens which make them more susceptible to activated immune cells. Through the development of next generation sequencing (NGS) technologies including whole genome sequencing (WGS) and RNAseq examining the mutational profile of tumors has become much easier. Analyzing the genetic signatures of tumors might identify patients who have higher chances to respond to checkpoint inhibition. Multiple studies have shown that a high tumor mutational burden (TMB) of greater than 100 non-synonymous single-nucleotide variants (nsSNV) per exome correlates with greater efficacy of diverse anti PDL1/PD-1 drugs [30].

The somatic mutation burden in bladder cancer is high, similar to other carcinogen-induced tumors. Only melanoma and non-small cell lung cancer have higher average point mutation burdens [31]. Rosenberg et al. studied mutations in 315 patients with urothelial carcinoma treated with Atezolizumab after chemotherapy failure. The median mutation load was significantly higher in responders than in non-responders assessed through focused genomic profiling of a 315-gene panel (12.4 vs 6.4 per mega base) to $\mathrm{p}<0.0001$. With the data obtained, the correlation of mutational load and response to atezolizumab was consistent with the pattern observed in other malignancies and reinforces the concept that the multiple mutations that occur in cancer create novel epitopes against which protective $\mathrm{T}$ cell responses are directed. It has been noted that TMB seems to have a threshold effect, with no appreciable therapeutic benefit until patients reach the top quartile of mutational burden [32]. At the moment the molecular testing platforms currently in use have a wide degree of variance in the gene panels used; hence, the harmonization of TMB across these platforms and the definition of the optimal threshold to define the high group that can be used for treatment selection are necessary next steps for the field [33].

It has been shown in colorectal cancer that mismatch repair deficiencies (MMRD) may prove to be a predictive biomarker for PDL1 immunotherapy. Patients with colorectal cancer and non-colorectal, MSI-derived cancers have significant response rates and prolonged survival when treated with pembrolizumab [34]. Notably, mutations in the mismatch repair enzyme $\mathrm{MSH} 2$ are specifically associated with both upper (5.6 \%) and lower tract (12.3\%) urothelial cancers [35]. Castro et al. documented the first case of characterized MMRD-urothelial cancer with a clinical benefit from PD-L1 immunotherapy. Follow-up clinical studies are required to determine the real predictive value of this promising biomarker in urothelial cancer.

\section{TCGA subtyping}

The Cancer Genome Atlas (TCGA) is an effort to describe genomic data in 33 types of cancer from more than 11,000 patients. This massive publicly available dataset has proven to be a treasure trove for biomarker discovery [36,37]. This dataset may also hold insights into predicting immunotherapy responses.

By analyzing the RNA-seq data from 129 tumors the collaboration identified four clusters (clusters I-IV) of urothelial bladder carcinoma [38]. The identification of these subtypes is clinically important because they have distinct prognoses and molecular mechanisms driving them. Therefore, it is possible that some subtypes may be appropriate for immune checkpoint therapy while others may benefit from traditional chemotherapy.

A handful of clinical trials have investigated the relationship that exists between the molecular subtype of bladder cancer and PD-1/PDL1 inhibition. The IMvigor 210 cohort 2 trial shows the greatest response to atezolizumab was observed in the luminal cluster II subtype [13]. In contrast, the Checkmate 275 study for nivolumab showed that it was basal subtype I that had the greatest response [14]. These discrepancies in response may be due to the variation in where the tissue was obtained. Both the IMVigor210 and Checkmate-275 cohorts allowed biopsy specimens from primary tumor, lymph nodes, or metastatic lesions for TCGA subtyping which may lead to inappropriate tumor classification. Additionally, the TCGA classification is not yet standardized which may be another source of discordance.

TCGA recently published the findings of their expanded bladder cancer study. In this updated study the researchers were able to subdivide the previously defined, major luminal and basal subtypes into luminal-papillary, luminal-infiltrated, luminal, basal-squamous subtypes and a lesser known, neuronal subtype [39]. Future clinical trials incorporating these more defined subtypes may provide evidence for TCGA subtyping as a reliable biomarker.

\section{Predictors of resistance}

\section{$J A K 1 / J A K 2$}

JAK1/2 are critical part of the interferon gamma (IFN $\gamma)$ pathway with STAT1 being downstream of that same signaling cascade. IFN $\gamma$ is produced by tumor-infiltrating lymphocytes and confer a functional advantage for tumor regression as it stimulates an immunosuppressive tumor microenvironment by increasing antigen presentation, PDL1 expression as well as anti-proliferative effect [40]. Zaretsky et al. identified JAK1 and JAK2 mutations in the relapse tumor of two patients with melanoma who were initially responsive to the PD-1 inhibitor pembrolizumab [41]. Although infiltrating CD8+ T cells can still recognize tumor cells and induce production of IFN $\gamma$, the tumor cells become considerably less responsive to the production of IFN $\gamma$ with a dysfunctional JAK1/2 mutation.

IFN $\gamma$ induced PD-L1 expression is essential as it has been reported to be associated with positive responses to PD-1/PD-L1 blockade in clinical studies $[42,43]$. Specifically, in the context of anti-PD-1 therapy, tumor cells with insensitivity to interferon gamma have a selective advantage. These tumors avoid the detrimental increase in antigen presentation and direct antiproliferative effects that are inducible by IFN $\gamma$. Studies are needed in the realm of bladder cancer as this can potentially be an important mechanism of acquired resistance. 


\section{Beta-2 microglobulin}

Zaretsky et al. also found a truncating mutation in the gene encoding Beta-2-microglobulin $(\beta 2 \mathrm{M})$ was identified that led to the loss of surface expression of MHC class I [41]. $\beta 2 \mathrm{M}$ is a small protein presenting in nearly all nucleated cells and most biological fluids, including serum, urine, and synovial fluid. $\beta 2 \mathrm{M}$ is crucial to stabilize cell surface MHC I, keep native structure of MHC I heavy chain, facilitate the binding of antigenic peptides, and generate additional high-affinity peptide-bindings [44]. Beta-2-microglobulin has a role in proper MHC class I fold and transport to the cell surface, and its deficiency has long been recognized as a genetic mechanism of acquired resistance to immunotherapy. Probing for this mutation in addition to JAK may be the building blocks for a panel to predict acquired resistance to immunotherapy.

\section{Predictors of hyperprogression}

\section{MDM2 family amplification}

While there are several biomarkers partially capable of predicting response and resistance, it may also be important to pursue biomarkers of hyper progressive disease (HPD). Hyper progressors are patients at risk of accelerated progression of their disease after immunotherapy. Champiat $e t$ al. were the first to define this feature in immunotherapytreated patients. They observed a total of $9 \%$ of patients experiencing $\mathrm{HPD}$, defined as a $>2$-fold increase of the tumor growth rate in patients with disease progression [45]. A recent study showed that specific genomic alterations such as the presence of MDM2 family amplification may be associated with accelerated progression [46]. They defined hyper-progression as time-to-treatment failure $<2$ months, $>50 \%$ increase in tumor burden compared to pre-immunotherapy imaging, and $>2$-fold increase in progression pace. In their paper they describe a 73-year-old man with metastatic bladder cancer to the liver who showed a $258 \%$ increase in the size of his liver masses 1.9 months after starting atezolizumab.

MDM2 amplification is found in about $7 \%$ of cancers and functions to inhibit the p53 tumor suppressor [47]. The exact mechanism linking MDM2 amplification and hyper-progression is unclear. These studies show that individuals with these alterations should be monitored closely if being treated with anti-PD1/PD-L1 agents. However larger studies and further validation is needed.

\section{Case report}

PD-L1 expression in bladder cancer circulating tumor cells (CTCs)

To demonstrate the potential of circulating tumor cells (CTCs) and staining of CTCs for PD-L1 as an additional novel biomarker. we have included a case report drawn from our recent experience. A 71-year old female presented with urinary retention and was later found to have a urethral mass invading into the deep muscle of her bladder, consistent with muscle-invasive urothelial carcinoma. Workup revealed no distant metastases. She was started on neoadjuvant chemotherapy with gemcitabine and cisplatin and then underwent a radical cystectomy with subsequent findings of tumor in her regional lymph nodes. She then completed adjuvant radiation therapy to the bladder. At the first follow-up six months after completion of radiation therapy, she had an MRI of her pelvis without findings of recurrent, residual, or metastatic disease in the pelvis.

Her next follow-up MRI one year later in the summer of 2017 however now showed new abdominal retroperitoneal and para-aortic

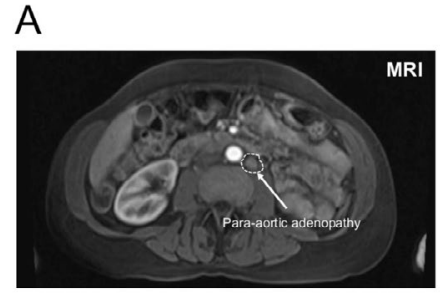

Before

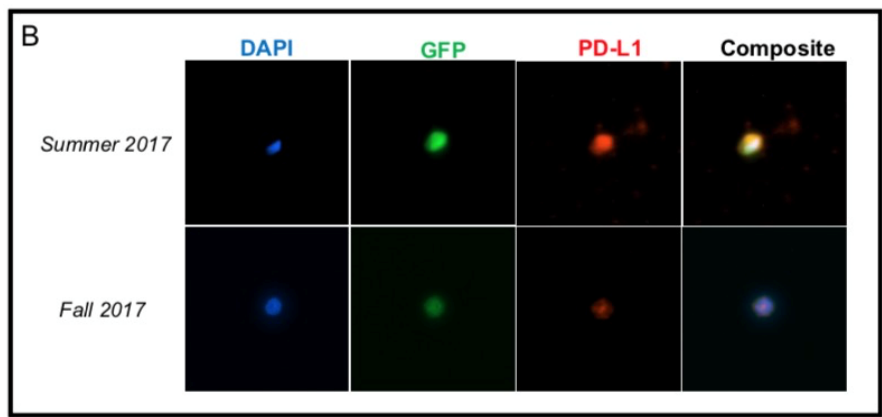

Figure 2. (A) Imaging of a patient with bladder cancer showing the emergence of paraaortic lymphadenopathy (arrow, MRI, "Before"). After 3 cycles of Pembrolizumab, the patient was noted to have significant reduction of the lymphadenopathy (CT, "After"). (B) Circulating tumor cells isolated from the patient before and after treatment stained positive for PD-L1 expression on immunofluorescence. The expression of green fluorescent protein (GFP) is driven by the elevated telomerase activity of the turmor and so identifies the CTCs. DAPI is a general nuclear stain that indicates intact cells

lymph nodes suspicious for metastatic disease (see Figure 2A, MRI, "Before" ). The tissue from the patient's original cystectomy was not available for re-analysis. However, she underwent circulating tumor cells (CTCs) analysis, which revealed 28 CTCs/per $\mathrm{ml}$ of blood. The CTCs were isolated from the patient's peripheral blood sample for immunofluorescence by a novel telomerase-based approach described previously [48] and the CTCs appeared to stain positive for PD-L1 (Figure 2B). The patient was then started on Pembrolizumab. The patient was noted to have significant reduction of lymphadenopathy following 3 cycles of Pembrolizumab (Figure 2A, CT, "After"), at which point CTC analysis revealed $21 \mathrm{CTC} / \mathrm{ml}$ but the CTCs were still PDL1 positive. After nine more cycles of pembrolizumab, the patient was noted to have had a complete response by imaging. She continues to do well with as yet no tumor recurrence.

\section{Conclusion}

The emergence of novel immunotherapies for bladder cancer represent major breakthroughs in the treatment of this disease. It is therefore pertinent to have reliable, standardized biomarkers to ensure the right therapy for the right patient. The current literature seems to suggest that no single biomarker will be sufficient in achieving that end. Rather, multiplexing of these biomarkers will most likely prove the most successful. Finally, we provide a glimpse into this possibility of composite biomarkers by demonstrating a case that combines PD-L1 expression with CTCs.

\section{Acknowledgement}

We are grateful to the Department of Radiation Oncology Clinical Research Coordinators and other members of the Kao Laboratory for superb technical assistance and support. The Research reported in this publication was supported by the National Center for Advancing Translational Sciences of the National Institutes of Health (NIH) under award number TL1TR001880 (for NS) and by the National Cancer Institute of the NIH under award number RO1CA201071. The content 
is solely the responsibility of the authors and does not necessarily represent the official views of the National Institutes of Health.

\section{References}

1. Siegel RL, Miller KD, Jemal A (2018) Cancer statistics CA. Cancer J Clin 68: 7-30.

2. Antoni S, Ferlay J, Soerjomataram I, Znaor A, Jemal A, et al. (2017) Bladder cancer incidence and mortality: a global overview and recent trends. Eur Urol 71: 96-108. [Crossref ]

3. Charlton ME, Adamo MP, Sun L, Deorah S (2014) Bladder cancer collaborative stage variables and their data quality, usage, and clinical implications: a review of SEER data, 2004-2010. Cancer 23: 3815-3825.

4. Ghasemzadeh A, Bivalacqua T, Hahn NM, Drake CG (2016) New strategies in bladder cancer: A second coming for immunotherapy. Clin Cancer Res 22: 793-801.

5. Kim J (2016) Immune checkpoint blockade therapy for bladder cancer treatment. Investig Clin Urol 57: S98-S105.

6. Farkona S, Diamandis EP (2016) Cancer immunotherapy: the beginning of the end of cancer? BMC Med 14: 73. [Crossref ]

7. Gakis (2014) The role of inflammation in bladder cancer. Adv Exp Med Biol 816: 183196. [Crossref ]

8. Patel SP, Kurzrock R (2015) PD-L1 Expression as a predictive biomarker in cancer immunotherapy. Mol Cancer Ther 14: 847-856. [Crossref ]

9. Nakanishi J, Wada Y, Matsumoto K, Azuma M, Kikuchi K, Ueda S, et al. (2007) Overexpression of B7-H1 (PD-L1) significantly associates with tumor grade and postoperative prognosis in human urothelial cancers. Cancer Immunol Immunother $56: 1173-1182$

10. Khunger M, Hernandez A V, Pasupuleti V (2017) Programmed cell death 1 (PD-1) ligand (PD-L1) expression in solid tumors as a predictive biomarker of benefit from pd-1/pd-11 axis inhibitors: A systematic review and meta-analysis. JCO Precis Oncol 3: $1-15$.

11. Powles T, O'Donnell PH, Massard C, Arkenau HT, Friedlander TW, et al. (2017) Efficacy and safety of durvalumab in locally advanced or metastatic urothelial carcinoma: updated results from a phase 1/2 open-label study. JAMA Oncol 3: e172411. [Crossref ]

12. Bellmunt J, de Wit R, Vaughn DJ, Fradet Y, Lee JL, et al. (2017) pembrolizumab as second-line therapy for advanced urothelial carcinoma. N Engl J Med 376: 1015-1026. [Crossref ]

13. Rosenberg JE, Hoffman-Censits J, Powles T (2016) Atezolizumab in patients with locally advanced and metastatic urothelial carcinoma who have progressed following treatment with platinum-based chemotherapy: a single-arm, multicentre, phase 2 trial. Lancet 387: 1909-1920.

14. Sharma P, Retz M, Siefker-Radtke A (2017) Nivolumab in metastatic urothelia carcinoma after platinum therapy (CheckMate 275): a multicentre, single-arm, phase 2 trial. Lancet Oncol 18: 312-322.

15. Hansen AR, Siu LL (2016) PD-L1 Testing in cancer. JAMA Oncol 2: 15.

16. Tretiakova M, Fulton R, Kocherginsky M (2017) Concordance study of PD-L1 expression in primary and metastatic bladder carcinomas: comparison of four commonly used antibodies and RNA expression. Mod Pathol.

17. Lau J, Cheung J, Navarro A, Lianoglou S, Haley B, et al. (2017) Tumour and host cell PD-L1 is required to mediate suppression of anti-tumour immunity in mice. Nat Commun 8: 14572. [Crossref]

18. Mahoney KM, Sun H, Liao X (2015) PD-L1 Antibodies to its cytoplasmic domain most clearly delineate cell membranes in immunohistochemical staining of tumor cells. Cancer Immunol Res 3: 1308-1315.

19. Pardoll DM (2012) The blockade of immune checkpoints in cancer immunotherapy. Nat Rev Cancer 12: 252-264. [Crossref]

20. Wang X, Teng F, Kong L, Yu J (2016) PD-L1 expression in human cancers and its association with clinical outcomes. Onco Targets Ther 9: 5023-5039. [Crossref ]

21. Jiang G, Huang C, Li J (2017) Role of STAT3 and FOXO1 in the divergent therapeutic responses of non-metastatic and metastatic bladder cancer cells to miR-145. Mol Cancer Ther 16: 924-935.

22. Zhu J, Li Y, Chen C (2017) NF- $\kappa$ B p65 Overexpression promotes bladder cancer cell migration via fbw7-mediated degradation of rhogdia protein. Neoplasia 19: 672-683.

23. Jin H, Yu Y, Hu Y (2015) Divergent behaviours and underlying mechanisms of cell migration and invasion in non-metastatic T24 and its metastatic derivative T24T bladder cancer cell lines. Oncotarget 6: 522-536.
24. Powell AA, Talasaz AH, Zhang H (2012) Single cell profiling of circulating tumor cells: transcriptional heterogeneity and diversity from breast cancer cell lines. Chin W-C, ed. PLoS One 7: e33788.

25. Liu X, Shin N, Koblish HK (2010) Selective inhibition of IDO1 effectively regulates mediators of antitumor immunity. Blood 115: 3520-3530.

26. Moon YW, Hajjar J, Hwu P, Naing A (2015) Targeting the indoleamine 2,3-dioxygenase pathway in cancer. J Immunother Cancer 3: 51. [Crossref ]

27. Smith D (2017) Epacadostat plus pembrolizumab in patients with advanced urothelial carcinoma: Preliminary phase I/II results of ECHO-202/KEYNOTE-037.

28. American Association for Cancer Research (2018) Blocking IDO1 helps shrink bladder, cervical tumors. Cancer Discov 8: OF3. [Crossref]

29. Chalmers ZR, Connelly CF, Fabrizio D (2017) Analysis of 100,000 human cancer genomes reveals the landscape of tumor mutational burden. Genome Med 9: 34

30. Jamieson NB, Maker AV (2017) Gene-expression profiling to predict responsiveness to immunotherapy. Cancer Gene Ther 24: 134-140.

31. Mouw KW (2017) DNA Repair pathway alterations in bladder cancer. Cancers (Basel) 9. [Crossref]

32. Aggen DH, Drake CG (2017) Biomarkers for immunotherapy in bladder cancer: a moving target. J Immunother Cancer 5: 94. [Crossref ]

33. Steuer CE, Ramalingam SS (2018) Tumor mutation burden: leading immunotherapy to the era of precision medicine? J Clin Oncol: JCO2017768770.

34. Le DT, Uram JN, Wang H, Bartlett BR, Kemberling H, et al. (2015) PD-1 Blockade in tumors with mismatch-repair deficiency. $N$ Engl J Med 372: 2509-2520. [Crossref ]

35. van der Post RS, Kiemeney LA, Ligtenberg MJL, et al. (2010) Risk of urothelial bladder cancer in Lynch syndrome is increased, in particular among MSH2 mutation carriers. JMed Genet 47: 464-470.

36. Wang B, Wan F, Sheng H, et al. (2018) Identification and validation of an 18-gene signature highly-predictive of bladder cancer metastasis. Sci Rep 8: 374. [Crossref]

37. Zhao F, Ge YZ, Zhou LH, Xu LW, Xu Z, et al. (2017) Identification of hub miRNA biomarkers for bladder cancer by weighted gene coexpression network analysis. Onco Targets Ther 10: 5551-5559. [Crossref ]

38. Weinstein JN, Akbani R, Broom BM (2014) Comprehensive molecular characterization of urothelial bladder carcinoma. Nature 507: 315-322.

39. Robertson AG, Kim J, Al-Ahmadie H (2017) Comprehensive molecular characterization of muscle-invasive bladder cancer. Cell 171: 540-556

40. Garcia-Diaz A, Shin DS, Moreno BH, Saco J, Escuin-Ordinas H, et al. (2017) Interferon receptor signaling pathways regulating PD-L1 and PD-L2 expression. Cell Rep 19: 1189-1201. [Crossref]

41. Shin DS, Zaretsky JM, Escuin-Ordinas H (2017) Primary resistance to PD-1 blockade mediated by JAK1/2 mutations. Cancer Discov 7: 1-56

42. Topalian SL, Taube JM, Anders RA, Pardoll DM. Mechanism-driven biomarkers to guide immune checkpoint blockade in cancer therapy. Nat Rev Cancer 16: 275-287.

43. Anagnostou V, Smith KN, Forde PM (2017) Evolution of neoantigen landscape during immune checkpoint blockade in non-small cell lung cancer. Cancer Discov 7: 1-3.

44. Li L, Dong M, Wang XG (2016) The implication and significance of beta 2 microglobulin: A conservative multifunctional regulator. Chin Med J (Engl).

45. Champiat S, Dercle L, Ammari S (2017) Hyperprogressive Disease is a new pattern of progression in cancer patients treated by anti-PD-1/PD-L1. Clin Cancer Res 23: 1920-1928.

46. Kato S, Goodman A, Walavalkar V, Barkauskas DA, Sharabi A, et al. (2017) Hyperprogressors after immunotherapy: analysis of genomic alterations associated with accelerated growth rate. Clin Cancer Res 23: 4242-4250.

47. Wade M, Li YC, Wahl GM (2013) MDM2, MDMX and p53 in oncogenesis and cancer therapy. Nat Rev Cancer 13: 83-96. [Crossref ]

48. Ju M, Kao GD, Steinmetz D (2014) Application of a telomerase-based circulating tumor cell (CTC) assay in bladder cancer patients receiving postoperative radiation therapy: a case study. Cancer Biol Ther 15: 683-687.

Copyright: (C)2019 Seranio N. This is an open-access article distributed under the terms of the Creative Commons Attribution License, which permits unrestricted use, distribution, and reproduction in any medium, provided the original author and source are credited. 